\title{
Can Farrell's allocative efficiency be generalized by the directional distance function approach?
}

\author{
Juan Aparicio ${ }^{\mathrm{a}, *}$, Jesus T. Pastor ${ }^{\mathrm{a}}$, Jose L. Zofio ${ }^{\mathrm{b}}$ \\ ${ }^{a}$ Center of Operations Research, Universidad Miguel Hernandez de Elche, E-03202 Elche, Alicante, Spain \\ ${ }^{\mathrm{b}}$ Departamento de Analisis Economico: Teoria Economica e Historia Economica, Universidad Autonoma de Madrid, E-28049 Madrid, Spain
}

\section{A R T I C L E I N F O}

\section{Article history:}

Received 21 February 2016

Accepted 3 August 2016

Available online 10 August 2016

\section{Keywords:}

Technical efficiency

Allocative efficiency

Directional distance functions

\begin{abstract}
A B S T R A C T
Cost or revenue efficiency measurement based on the approach initiated by Farrell has received great attention from academics and practitioners since the fifties. Farrell's approach decomposes cost efficiency into two different sources, viz. technical efficiency and allocative efficiency. Technical efficiency is estimated by means of the Shephard input or output distance functions, while allocative efficiency is derived as a residual between cost or revenue efficiency and its corresponding technical efficiency component. The directional distance function $(D D F)$ was introduced later in the literature to complete duality theory with respect to the profit function and as a generalization of the Shephard input and output distance functions. Considering the case of cost efficiency we show that, although the DDF correctly encompasses the technical efficiency component of the Farrell approach, this is not true for the allocative component. We show that both approaches yield different allocative (in)efficiency terms - unless technical efficiency is assumed, and how these terms are related. The practical implications of the multiplicative and additive approaches are discussed and illustrated.
\end{abstract}

(c) 2016 Elsevier B.V. All rights reserved.

\section{Introduction}

Cost or revenue efficiency measurement based on the approach initiated by Farrell (1957) has received great attention from academics and practitioners. Since Farrell, researchers have analytically decomposed cost and revenue efficiency into technical efficiency and allocative efficiency. In the spirit of this decomposition, technical efficiency is first of all estimated resorting to radial movements, relating this particular component to both Debreuś (1951) coefficient of resource utilization and the inverse of Shephard's input or output distance functions (Shephard, 1953). Secondly, in accordance with Farrell's approach, allocative efficiency is derived as a residual between cost or revenue efficiency and its corresponding technical efficiency component. As a result of this residual nature, the mathematical formulation of the estimator of the allocative efficiency component has received much less attention in the literature than that associated with technical efficiency.

While Farrell resorted to Shephard's distance function for his decomposition, nowadays there are alternative ways of estimating technical efficiency, e.g., the generalized distance function by

\footnotetext{
* Corresponding author. Fax: +34 966658715.

E-mail address: j.aparicio@umh.es (J. Aparicio).
}

Chavas and Cox (1999), the Hölder distance function by Briec (1998) and the directional distance function (DDF) by Chambers, Chung, and Färe (1996a, 1998). In particular, the precursor of the latter is the benefit function, a notion introduced by Luenberger (1992) in consumer theory as a generalization of the willingnessto-pay concept, measured with respect to an arbitrary bundle of goods. Luenberger (1992) also introduced the shortage function, which is the analog for production theory of the benefit function defined for consumer theory. Later, Chambers et al. (1996a, 1998) renamed the shortage function as the directional distance function, $D D F$, highlighting its properties as distance function and its geometrical interpretation.

In recent times, overall inefficiency has been recurrently decomposed by using duality theory (Zalinescu, 2010). Examples of this are Färe, Grosskopf, and Zaim (2002), where the hyperbolic measure was used to decompose the Georgescu-Roegen return to the dollar measure, Cooper, Pastor, Aparicio, and Borras (2011) and Aparicio, Borras, Pastor, and Vidal (2013a), who determined the existing relationship between the profit function and the weighted additive measure, Aparicio, Pastor, and Ray (2013b), who proved that the lost profit on outlay may be decomposed through a modified directional distance function, Aparicio, Borras, Pastor, and Vidal (2015a), where it is shown that cost (revenue) inefficiency can be decomposed resorting to the input (output) Russell measure and, finally, Färe, Fukuyama, Grosskopf, and Zelenyuk (2015), 
who showed how profit inefficiency is related to the slack-based directional distance function.

Focusing on the input side, Chambers et al. (1996a) introduced the directional input distance function, which measures the amount that one can translate an 'observed' input vector from itself to the frontier of the technology in a pre-assigned reference direction vector. Additionally, when the reference direction is considered equivalent to the assessed input vector, the directional input distance function encompasses the Shephard input distance function. In this way, the directional input distance function constitutes the analytical tool that allows breaking the straight jacket represented by the classical and restrictive framework of the radial based decomposition of cost efficiency (Farrell, 1957) and extends it to the case of considering any possible direction in the input space. Specifically, Färe and Grosskopf $(2000,2003)$ show that cost inefficiency may be additively decomposed into technical inefficiency plus allocative inefficiency by using the directional input distance function. These features, among others, have yielded an increasing interest of researchers in the $D D F$ in the last two decades.

In this paper we show that, in contrast to what is commonly assumed, Farrell's classical decomposition of cost efficiency is not completely encompassed by the approach based on the directional input distance function, except for the set of technically efficient observations, which is trivial. We also show that the Farrell and $D D F$ represent alternative approaches that yield different cost (in)efficiency decompositions. Therefore, practitioners must be aware that their choice of modeling framework is not neutral, neither in relation to the relative values of the technical and allocative terms nor the reference benchmarks, which may result in wrong interpretations and lead to faulty managerial prescriptions.

In Section 2 we present some preliminary notions and wellknown results regarding the decomposition of cost efficiency into its technical and allocative components, for both the Farrell and $D D F$ approaches. Section 3 shows that, contrary to current belief, the $D D F$ approach does not generalize the Farrell approach when the directional vector corresponds to the observed input vector; particularly when both approaches yield alternative definitions of the allocative inefficiency term. Here, we show the existing relationship between both approaches and highlight their differences and alternative interpretations. Conclusions are drawn in Section 4.

\section{Preliminary notions, results and notation}

In this section, we formalize some key notions about the technology and distance functions and recall how cost efficiency may be decomposed.

A technology is a set $T \subset R_{+}^{m} \times R_{+}^{s}$ satisfying several axioms; the most usual are the following (Färe \& Primont, 1995): (A1) $T$ is closed, (A2) inputs and outputs are freely disposable, i.e. $(\mathbf{x}, \mathbf{y}) \in T$, $\left(\mathbf{x}^{\prime}, \mathbf{y}^{\prime}\right) \in R_{+}^{m} \times R_{+}^{s}$ and $\mathbf{x}^{\prime} \geq \mathbf{x}, \mathbf{y}^{\prime} \leq \mathbf{y}$ imply $\left(\mathbf{x}^{\prime}, \mathbf{y}^{\prime}\right) \in T$, (A3) there is no free lunch, i.e., $(\mathbf{0}, \mathbf{y}) \in T$ implies $\mathbf{y}=\mathbf{0}$, (A4) doing nothing is feasible, i.e. $(\mathbf{0}, \mathbf{0}) \in T$ and (A5) $T$ is convex.

For the sake of brevity, we state our discussion in the input space, defining the input requirement set $L(\mathbf{y})$ as the set of nonnegative inputs $\mathbf{x} \in R_{+}^{m}$ that can produce non-negative output $\mathbf{y} \in$ $R_{+}^{s}$, formally $L(\mathbf{y})=\left\{\mathbf{x} \in R_{+}^{m}:(\mathbf{x}, \mathbf{y}) \in T\right\}$, and the isoquant of $L(\mathbf{y})$ : $\operatorname{IsoqL}(y)=\{x \in L(y): \varepsilon<1 \Rightarrow \varepsilon x \notin L(y)\}$.

Let us also denote by $C(\mathbf{y}, \mathbf{w})$ the minimum cost of producing the output level $\mathbf{y}$ given the input market price vector $\mathbf{w}=$ $\left(w_{1}, \ldots, w_{m}\right) \in R_{++}^{m}: C(\mathbf{y}, \mathbf{w})=\min \left\{\sum_{i=1}^{m} w_{i} x_{i}: \mathbf{x} \in L(\mathbf{y})\right\}$.

The standard (multiplicative) Farrell approach (Farrell, 1957) views cost efficiency $(C E)$ as originating from technical efficiency $(T E)$ and allocative efficiency $(A E)$. Specifically, Farrell quantified, and therefore defined each of these terms as follows:

$\underbrace{\frac{C(\mathbf{y}, \mathbf{w})}{C(\mathbf{x})}}_{C E}=\underbrace{\frac{1}{D_{i}(\mathbf{y}, \mathbf{x})}}_{T E} \cdot A E$,

where $\quad C(\mathbf{x})=\sum_{i=1}^{m} w_{i} x_{i} \quad$ is the cost at $\mathbf{x}, \quad D_{i}(\mathbf{y}, \mathbf{x})=$ $\sup \{\delta>0: \mathbf{x} / \delta \in L(\mathbf{y})\}$ is the Shephard input distance function (Shephard, 1953) and $A E$ is defined residually as $A E=C E / T E$.

After Farrell's work, and particularly over the last two decades, part of the literature has focused on duality theory and distance functions so as to provide a consistent theoretical framework for the cost efficiency decomposition (see Chambers et al., 1996a; Briec, 1997; Briec \& Lesourd, 1999). In particular, regarding the directional input distance function, let $\mathbf{g}=\left(g_{1}, \ldots, g_{m}\right) \in R_{+}^{m}$ be a vector such that $\mathbf{g} \neq \mathbf{0}$, then the directional input distance function is defined as $\vec{D}_{i}(\mathbf{x}, \mathbf{y} ; \mathbf{g})=\sup \{\beta: \mathbf{x}-\beta \mathbf{g} \in L(\mathbf{y})\}$. Färe and Grosskopf (2000, 2003, p. 25, expression 1.44) showed that the directional cost inefficiency $(D C I)$ may be additively decomposed into technical inefficiency (DTI) plus allocative inefficiency (DAI) relying on the directional input distance function and duality results:

$\underbrace{\frac{\sum_{i=1}^{m} w_{i} x_{i}-C(\mathbf{y}, \mathbf{w})}{\sum_{i=1}^{m} w_{i} g_{i}}}_{D C I(\mathbf{g})}=\underbrace{\overrightarrow{D_{i}}(\mathbf{x}, \mathbf{y} ; \mathbf{g})}_{D T I(\mathbf{g})}+D A I(\mathbf{g})$,

where $\operatorname{DAI}(\mathbf{g})$ is defined residually as $\operatorname{DAI}(\mathbf{g})=\operatorname{DCI}(\mathbf{g})-\operatorname{DTI}(\mathbf{g})$.

\section{The results}

Since Färe and Grosskopf (1997), it is commonly accepted that the directional distance function encompasses the Farrell approach for decomposing cost efficiency. Indeed, if $\mathbf{g}=\mathbf{x}$ then $\vec{D}_{i}(\mathbf{x}, \mathbf{y} ; \mathbf{x})=$ $1-1 / D_{i}(\mathbf{x}, \mathbf{y})$, and from this relationship the directional input distance function, which measures technical inefficiency, encompasses the Shephard input distance function in the input space. Therefore, $\operatorname{DTI}(\mathbf{x})=1-T E$, and it is natural to extrapolate this relationship to the cost and allocative measures: $D C I(\mathbf{x})=1-C E$ and $\operatorname{DAI}(\mathbf{x})=1-A E$.

However, the directional and Farrell approaches are not completely equivalent since the allocative components in (1) and (2) do not verify the appropriate mathematical relationship, something that does hold in the case of cost and technical terms. To show that, let us explicitly rewrite $D T I(\mathbf{g})$ in (2) as $\vec{D}_{i}(\mathbf{x}, \mathbf{y} ; \mathbf{g})=\left(\sum_{i=1}^{m} w_{i} \vec{D}_{i}(\mathbf{x}, \mathbf{y} ; \mathbf{g}) g_{i}\right) / \sum_{i=1}^{m} w_{i} g_{i}$. Then, from the relationship $\operatorname{DAI}(\mathbf{g})=D C I(\mathbf{g})-\operatorname{DTI}(\mathbf{g}), \operatorname{DAI}(\mathbf{g})$ can be expressed as $\left[\sum_{i=1}^{m} w_{i}\left(x_{i}-\vec{D}_{i}(\mathbf{x}, \mathbf{y} ; \mathbf{g}) g_{i}\right)-C(\mathbf{y}, \mathbf{w})\right] / \sum_{i=1}^{m} w_{i} g_{i}$. Now, considering $\mathbf{g}=\mathbf{x}$ we have that:

$$
\operatorname{DCI}(\mathbf{x})=\frac{\sum_{i=1}^{m} w_{i} x_{i}-C(\mathbf{y}, \mathbf{w})}{\sum_{i=1}^{m} w_{i} x_{i}}=1-\frac{C(\mathbf{y}, \mathbf{w})}{C(\mathbf{x})}=1-C E,
$$

and

$$
\operatorname{DTI}(\mathbf{x})=\vec{D}_{i}(\mathbf{x}, \mathbf{y} ; \mathbf{x})=1-1 / D_{i}(\mathbf{x}, \mathbf{y})=1-T E .
$$

However, if $\mathbf{g}=\mathbf{x}$, regarding the allocative inefficiency term in (2), we have that $\operatorname{DAI}(\mathbf{x})=\frac{\sum_{i=1}^{m} w_{i}\left(x_{i}-\vec{D}_{i}(\mathbf{x}, \mathbf{y} ; \mathbf{x}) x_{i}\right)-C(\mathbf{y}, \mathbf{w})}{\sum_{i=1}^{m} w_{i} x_{i}}=$ $\frac{\sum_{i=1}^{m} w_{i}\left(x_{i}-\left(1-1 / D_{i}(\mathbf{x}, \mathbf{y})\right) x_{i}\right)-C(\mathbf{y}, \mathbf{w})}{\sum_{i=1}^{m} w_{i} x_{i}}=\frac{1}{D_{i}(\mathbf{y}, \mathbf{x})}-\frac{C(\mathbf{y}, \mathbf{w})}{C(\mathbf{x})}=\frac{1}{D_{i}(\mathbf{y}, \mathbf{x})}(1-A E)$, since by (1) $1-A E=1-D_{i}(\mathbf{y}, \mathbf{x})\left(\frac{C(\mathbf{y}, \mathbf{w})}{C(\mathbf{x})}\right)$. Therefore, $D A I \quad(\mathbf{x}) \neq A I=1-A E$ except for the case in which $D_{i}(\mathbf{y}, \mathbf{x})=1$. In other words, the allocative inefficiency estimated from (2) does not present the desired relationship: one minus the allocative efficiency estimated from (1). This fact has important implications when cost efficiency or inefficiency must be decomposed for technically inefficient firms by resorting to the traditional Farrell approach or to the 


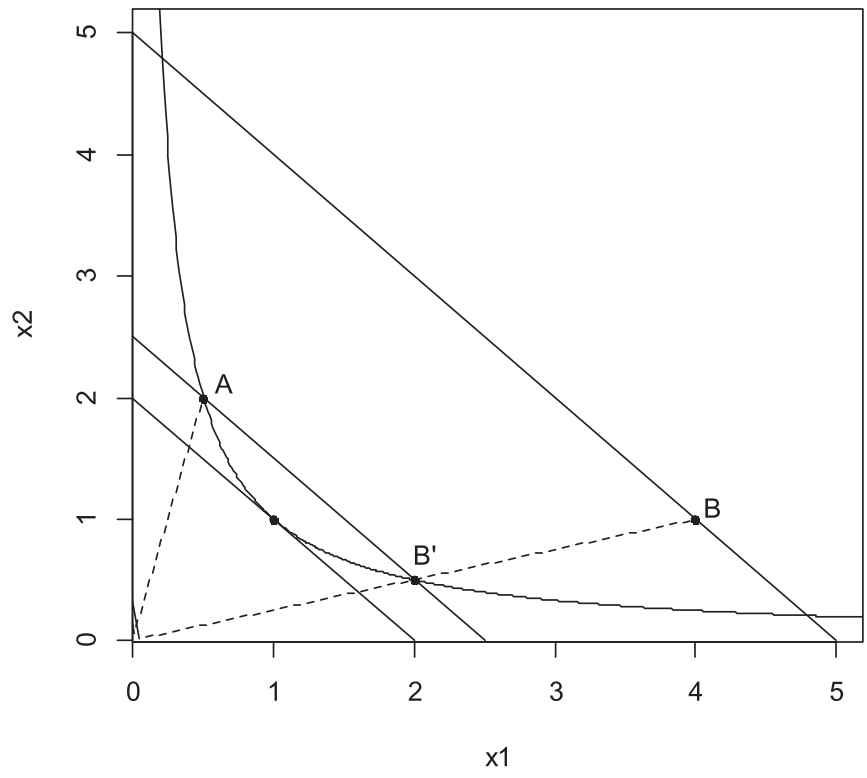

Fig. 1. Graphical example.

directional input distance function approach, even in the case of selecting $\mathbf{g}=\mathbf{x}$. We illustrate this situation through Fig. 1.

Let us assume that $L(y)=\left\{\mathbf{x} \in R_{+}^{2}: y=x_{1}{ }^{1 / 2} x_{2}{ }^{1 / 2}\right\}$ with $y \geq 0$. We also suppose that we evaluate the cost efficiency and its sources corresponding to points $A=(0.5,2 ; 1)$ and $B=(4,1 ; 1)$. Additionally, we assume that $w_{1}=w_{2}=1$. In this context, the Shephard input distance functions for $A$ and $B$ are $D_{i}(A)=1$ and $D_{i}(B)=2$, respectively. Furthermore, by applying (1) we have that $A E_{A}=A E_{B}=2 / 2.5=0.8$ in the Farrell approach, i.e., $A$ and $B$ present the same allocative efficiency level. In contrast, if we resort to the directional input distance function, we have that $D A I_{A}$ $(\mathbf{x})=\frac{0.5}{2.5}=(1-0.8)=0.2>\frac{0.5}{5}=\frac{1}{2}(1-0.8)=0.1=D A I_{B}$. In this way, we show that the Farrell and directional input distance functions do not always coincide in the magnitude and order relationship for the allocative (in)efficiency component. For firm $B$, multiplicative (Farrell) cost efficiency decomposes as follows: $0.4=0.5$ - 0.8 , while the additive $(D D F)$ cost inefficiency is $0.6=0.5+0.1$. Therefore, in the situation described in Fig. 1, Farrell's approach indicates that $A$ and $B$ have the same allocative efficiency. However, the DDF approach does not yield the same result.

The attractiveness of the Farrell approach in defining cost efficiency as the ratio of minimum cost to observed cost, is that the technical efficiency corresponding to the inverse of Shephard's distance function, measures proportional cost savings in the quantity $T I=1-T E=1-1 / D_{i}(\mathbf{y}, \mathbf{x})$, resulting from the reduction in input usage - graphically corresponding the projection of the observed firm to the technical efficient benchmark; e.g., $B$ to $B^{\prime}$ in Fig. 1 . This projection along the ray-vector passing through the origin constitutes a natural direction that keeps input proportions - input-mix - constant, and yields allocative efficiency as a residual that corresponds to the ratio of minimum cost to the cost at the technical efficient projection. Therefore all firms situated along the ray vector exhibit the same allocative efficiency value, i.e., that corresponding to their projection $B^{\prime}$.

For the $D D F$ approach, its additive nature prevents the complete generalization of the Farrell decomposition to the allocative term when setting $\mathbf{g}=\mathbf{x}$. In this case, the technical efficiency term $\vec{D}_{i}(\mathbf{x}, \mathbf{y} ; \mathbf{x})$ measures the projection to the same reference benchmark $B^{\prime}$, and while cost and technical inefficiency can be related to the multiplicative Farrell terms, these transformations result in an allocative efficiency residual that embeds the technical efficiency value TE. This implies that the directional allocative inefficiency value numerically depends on the specific technical efficiency of the firm under evaluation, i.e. $D A I(\mathbf{x})=T E(1-A E)$. Therefore, the firms situated along the ray vector stop exhibiting the allocative inefficiency value corresponding to the technically efficient projection, as allocative inefficiency, defined as the complement to the Farrell's term, $A I=(1-A E)$, needs to be rescaled by the technical efficiency value $T E$ so as to close the additive relationship.

However, as the following proposition shows, the DDF decomposition exhibits other properties that are attractive in the additive framework. While in the Farrell approach, two firms present the same allocative efficiency if they belong to the same ray vector passing through the origin - regardless of their technical efficiency and observed cost, the flexibility of the directional distance function renders the technical inefficiency term dependent on $\mathbf{g}$, passing on to the residual allocative inefficiency term that closes up the decomposition. For example, in Fig. 1 it would be possible to project $B$ to a point different from $B^{\prime}$, i.e., $\mathbf{g} \neq \mathbf{X}$, with associated technical efficiency and residual allocative efficiency values completely unrelated to the Farrell decomposition. However, for $\mathbf{g}=\mathbf{x}$, two firms present the same allocative inefficiency if they belong to the same isocost line - regardless, once again of their technical inefficiency. This result is summarized in the next proposition establishing the conditions for which allocative inefficiency is equal across firms, as in the case of the Farrell approach.

Proposition 1. Let $\mathbf{x}_{A}, \mathbf{x}_{B} \in L(\mathbf{y})$ and $A E_{A}=A E_{B}$. Then, $D A I_{A}\left(\mathbf{x}_{A}\right)=$ $D_{B} I_{B}\left(\mathbf{x}_{B}\right)$ is equivalent to $C\left(x_{A}\right)=C\left(x_{B}\right)$.

Proof. By $\operatorname{DAI}(\mathbf{x})=\frac{1}{D_{i}(\mathbf{y}, \mathbf{x})}(1-A E)=T E \cdot(1-A E)$ and the hypothesis of the proposition, we have that $D_{A} I_{A}\left(\mathbf{x}_{A}\right)=D A I_{B}\left(\mathbf{x}_{B}\right) \Leftrightarrow$ $T E_{A}=T E_{B} \Leftrightarrow C E_{A}=C E_{B} \Leftrightarrow C\left(x_{A}\right)=C\left(x_{B}\right)$.

It is worth mentioning that in the numerical example illustrated in Fig. 1, points $A$ and $B$ do not belong to the same isocost line and exhibit different technical efficiencies, and therefore by Proposition 1 the allocative inefficiencies estimated by the $D D F$ approach cannot be the same, even in the case of $A E_{A}=A E_{B}$.

Therefore, the multiplicative (Farrell) and additive (DDF) approaches to decompose cost (in)efficiency represent two alternative mathematical definitions, which can be related through Proposition 1, but offer different decomposition results. As the Farrell approach is well established among practitioners and even implemented in software packages, it could be considered as criterion for a preferred approach - particularly since most applications using the directional input distance functions set $\mathbf{g}=\mathbf{x}$ - and therefore the directional allocative component in (2) would underestimate allocative inefficiency for technically inefficient firms, since $\operatorname{DAI}(\mathbf{x})=\frac{1}{D_{i}(\mathbf{y}, \mathbf{x})}(1-A E)$ and $\frac{1}{D_{i}(\mathbf{y}, \mathbf{x})} \in(0,1]$ for $\mathbf{y} \neq \mathbf{0}$. However, the opposite could also be argued if the reference benchmark were the directional approach, with the Farrell decomposition overstating allocative efficiency. In the end, it all depends on the choice of the directional vector, as both decompositions can only be related if $\mathbf{g}=\mathbf{x}$.

To show the magnitude of the relative over and under estimations under the alternative multiplicative and additive approaches; i.e., the numerical divergence between the allocative efficiency term a la Farrell $(A E)$ and directional allocative inefficiency $D A I(\mathbf{x})$, we define and systematically study the difference between these two terms. Knowing that a firm is allocatively efficient in the sense of Farrell when $A E=1$, and therefore its directional allocative inefficiency is $\operatorname{DAI}(\mathbf{x})=0$, we recall once again Farrell's allocative inefficiency: $A I=(1-A E)$. This simple transformation makes both measures comparable in value, i.e., both approaches measure inefficiency. 
Table 1

Divergence between allocative (in)efficiencies: $f(A E, T E)=A I-D A I(\mathbf{x})$.

\begin{tabular}{|c|c|c|c|c|c|c|c|c|c|c|c|c|}
\hline & & \multicolumn{11}{|l|}{$T E$} \\
\hline & & 0 & 0.1 & 0.2 & 0.3 & 0.4 & 0.5 & 0.6 & 0.7 & 0.8 & 0.9 & 1 \\
\hline \multirow[t]{11}{*}{$A E$} & 0 & 1.000 & 0.900 & 0.800 & 0.700 & 0.600 & 0.500 & 0.400 & 0.300 & 0.200 & 0.100 & 0.000 \\
\hline & 0.1 & 0.900 & 0.810 & 0.720 & 0.630 & 0.540 & 0.450 & 0.360 & 0.270 & 0.180 & 0.090 & 0.000 \\
\hline & 0.2 & 0.800 & 0.720 & 0.640 & 0.560 & 0.480 & 0.400 & 0.320 & 0.240 & 0.160 & 0.080 & 0.000 \\
\hline & 0.3 & 0.700 & 0.630 & 0.560 & 0.490 & 0.420 & 0.350 & 0.280 & 0.210 & 0.140 & 0.070 & 0.000 \\
\hline & 0.4 & 0.600 & 0.540 & 0.480 & 0.420 & 0.360 & 0.300 & 0.240 & 0.180 & 0.120 & 0.060 & 0.000 \\
\hline & 0.5 & 0.500 & 0.450 & 0.400 & 0.350 & 0.300 & 0.250 & 0.200 & 0.150 & 0.100 & 0.050 & 0.000 \\
\hline & 0.6 & 0.400 & 0.360 & 0.320 & 0.280 & 0.240 & 0.200 & 0.160 & 0.120 & 0.080 & 0.040 & 0.000 \\
\hline & 0.7 & 0.300 & 0.270 & 0.240 & 0.210 & 0.180 & 0.150 & 0.120 & 0.090 & 0.060 & 0.030 & 0.000 \\
\hline & 0.8 & 0.200 & 0.180 & 0.160 & 0.140 & 0.120 & 0.100 & 0.080 & 0.060 & 0.040 & 0.020 & 0.000 \\
\hline & 0.9 & 0.100 & 0.090 & 0.080 & 0.070 & 0.060 & 0.050 & 0.040 & 0.030 & 0.020 & 0.010 & 0.000 \\
\hline & 1 & 0.000 & 0.000 & 0.000 & 0.000 & 0.000 & 0.000 & 0.000 & 0.000 & 0.000 & 0.000 & 0.000 \\
\hline
\end{tabular}

Let us define the difference between Farrell's allocative (in)efficiency $A I$ and the directional allocative inefficiency, $D A I(\mathbf{x})$, as follows: $f(A E, T E)=A I-D A I(\mathbf{x})=(1-A E)-T E(1-A E)=(1-$ $A E)(1-T E)$, since $\operatorname{DAI}(\mathbf{x})=T E(1-A E)$. Consequently, when $f(A E$, $T E)=0$, allocative (in)efficiency is the same in both approaches and there is no divergence between the two; e.g., $A E=1$ and $D A I(\mathbf{x})=0$. The image of $f$ is $[0,1)$ since $A E$ and $T E$ take values in the range $(0,1]$. This facilitates the comparison between the two in terms of the familiar Farrell efficiencies and allows us to discuss key values, which are summarized in Table 1 presenting the divergence between the (in)efficiency measures for alternative values of $A E$ and TE.

Therefore, both approaches yield equivalent categorization of a firm as allocatively efficient: $f(A E, T E)=A I-D A I(\mathbf{x})=0$, in the case that $A E=1$, regardless of its technical efficiency (and vice versa for $T E=1$ ), or both as completely efficient in allocative and technical terms $(A E=1$ and $T E=1)$. For any other pair of efficiency values $(0<A E<1,0<T E<1)$, both diverge numerically, with their difference increasing as the firm is more inefficient, and approximating the maximum value (one) in the limit case that the firm is completely inefficient in technical and allocative terms $(A E \rightarrow 0$ and $T E \rightarrow 0){ }^{1}$ Figs. 2 and 3 allow us to visualize the values presented in Table 1 through a set of contour (level) curves, and the three dimensional plot of the function, respectively. The properties of the function can be explored through its partial derivatives: $d f / d A E=T E-1$ and $d f / d T E=A E-1$. These linear functions show that the discrepancy between both measures is linearly decreasing in the technical and allocative efficiency values.

The same discussion between the two worlds (multiplicative vs additive, and their eventual divergence) can be found in the literature regarding the comparison of the traditional Malmquist productivity index, which is based on Shephard's distance function (Caves, Christensen, \& Diewert, 1982) and is multiplicative in nature, with the more recent Luenberger productivity indicator based on the directional distance function (Chambers, Färe, \& Grosskopf, 1996b), and which is additive in nature. In fact, studying the gap between Malmquist indexes and Luenberger indicators has been the focus of recent research. Boussemart, Briec, Kerstens, and Poutineau (2003), under the constant returns to scale assumption, relate the Luenberger indicator, based on the proportional directional distance function, with the Malmquist index, based on Shephard's input distance function, and report, by means of an example, that the Malmquist index measures twice as much productivity change as the Luenberger indicator. They further re-

\footnotetext{
${ }^{1}$ It is also possible to express the difference between both allocative inefficiencies in relative (percentage) terms. Let us define $g(\mathrm{AE}, T E)=100 \bullet([A I-$ $\operatorname{DAI}(\mathbf{x})] / A I) \%=100 \bullet(1-T E) \%$. When $T E=1, \operatorname{DAI}(\mathbf{x})=(1-\mathrm{AE})$ with $g(\mathrm{AE}, T E)=0 \%$, while if $T E<1$ then the difference increases in $T E$ and approximates its maximum value as $T E \rightarrow 0$.
}

late certain Malmquist indexes with certain Luenberger indicators by means of first and second order approximations. Balk, Färe, Grosskopf, and Margaritis (2008), assuming strong disposability of outputs and efficiency at each time period, establish an exact relationship between the Malmquist index and the Luenberger indicator both based on output distance functions. Finally, Briec, Kerstens, and Peypoch (2012) revise the last mentioned paper and point out that the relation proposed is not between Malmquist and Luenberger but between Malmquist and the two components of Luenberger. They also provide a new exact relationship under the same assumptions of Balk et al. (2008), and prove a specific equality between the logarithm of the output-oriented Malmquist productivity index and the output-oriented Luenberger indicator, resorting to the unit directional vector on the output side and defined on the logarithm of the data. ${ }^{2}$

As happens in this paper, Boussemart et al. (2003), Balk et al. (2008) and Briec et al. (2012) did not side with any of the two approaches against the other one (Malmquist index vs Luenberger indicator), but these works did serve to point out to practitioners the non-equivalence and the existence of a certain relationship between the two methodologies.

Although both methods, the Farrell and DDF approaches, for measuring and decomposing cost inefficiency are valid, we would like to highlight some interesting features of each in order to provide practitioners with different criteria for selecting one or the other. In this way, one remarkable property of the Farrell approach is that allocative efficiency always coincides with the allocative efficiency of the projection point on the corresponding isoquant. Let us formally establish this statement as follows.

Proposition 2. Let $\mathbf{x}_{A} \in L(\mathbf{y})$ and $\mathbf{x}_{B} \in \operatorname{IsoqL}(\mathbf{y})$ such that $\mathbf{x}_{B}=\rho \mathbf{x}_{A}$ and $D_{i}\left(\mathbf{y}, \mathbf{x}_{B}\right)=1$. Then, $A E_{A}=A E_{B}$.

Proof. Under these hypotheses, $\rho=\left(D_{i}\left(\mathbf{y}, \mathbf{x}_{A}\right)\right)^{-1}$. By (1) and $D_{i}\left(\mathbf{y}, \mathbf{x}_{B}\right)=1$, we have that $A E_{B}=\frac{C(\mathbf{y}, \mathbf{w})}{C\left(\mathbf{x}_{B}\right)}=\frac{C(\mathbf{y}, \mathbf{w})}{C\left(\rho \mathbf{x}_{A}\right)}=\frac{1}{\rho} \frac{C(\mathbf{y}, \mathbf{w})}{C\left(\mathbf{x}_{A}\right)}=$ $D_{i}\left(\mathbf{y}, \mathbf{x}_{A}\right) \frac{C(\mathbf{y}, \mathbf{w})}{C\left(\mathbf{x}_{A}\right)}=A E_{A}$.

Proposition 2 means that Farrell's approach preserves the value of allocative efficiency along the contracting path given by the input mix. As Aparicio, Pastor, and Zofio (2015b) remark, this is a desirable property for a suitable decomposition of economic efficiency since the allocative efficiency of the firm remains unchanged in the process of gaining technical efficiency by reducing the observed input vector. Only in this way it is possible to be sure that the distance function measures 'pure' technical efficiency instead of a mix of technical and allocative efficiency. However, the

\footnotetext{
${ }^{2}$ Unfortunately, we were not able to establish an exact relationship in our context between both decompositions following the same mathematical structure developed by Briec et al. (2012).
} 


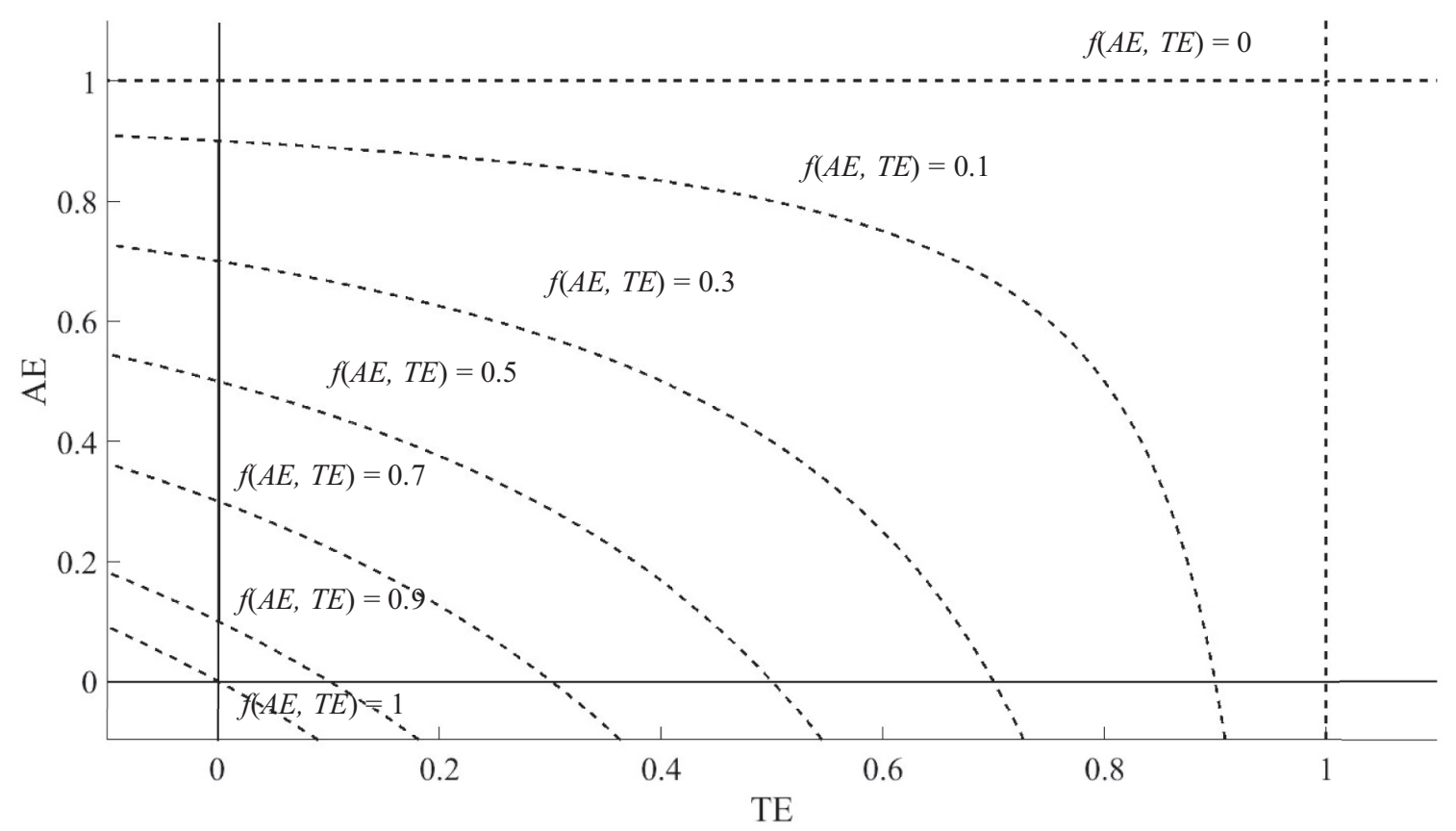

Fig. 2. Contour curves of $f(A E, T E)=A I-D A I(\mathbf{x})$.

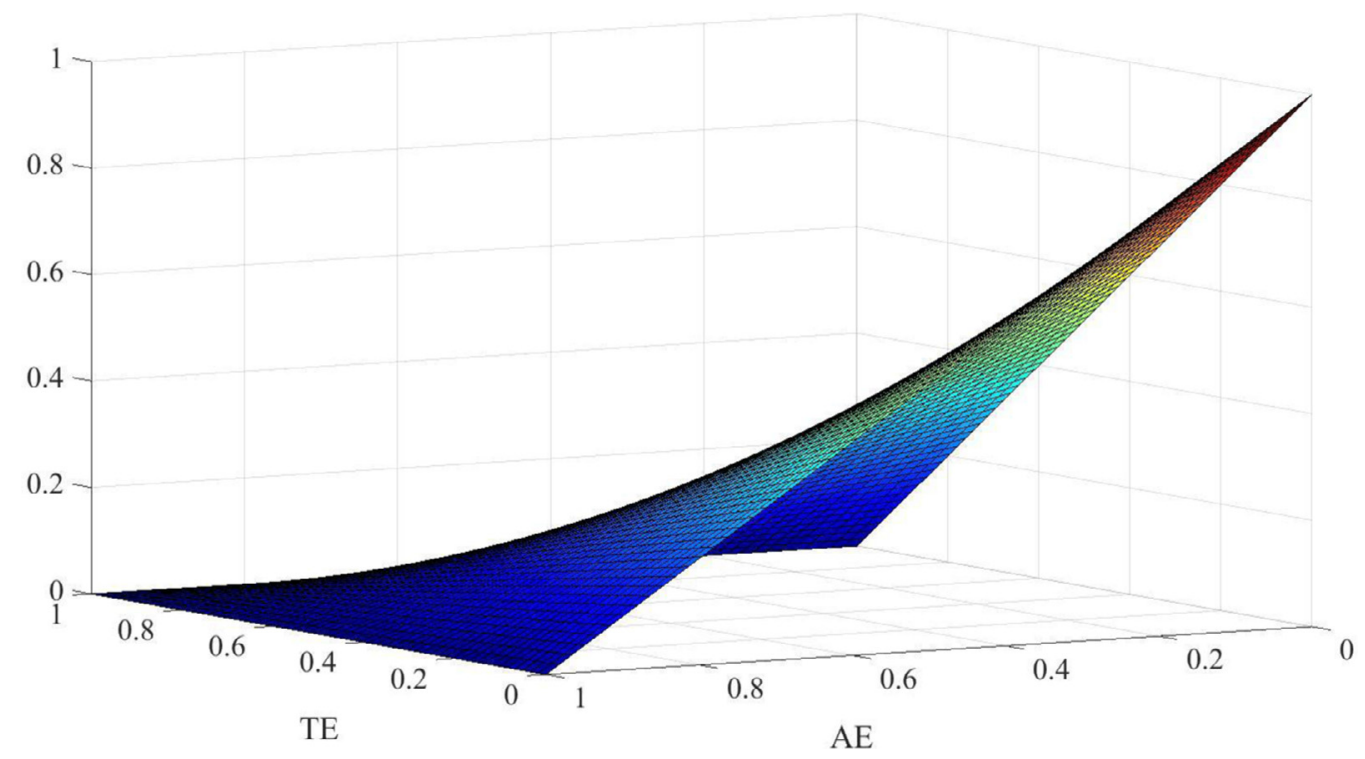

Fig. 3. Three-dimensional plot of $f(A E, T E)=A I-D A I(\mathbf{x})$.

directional distance function approach does not meet this property in general. In this case, for example, the result is satisfied under the assumption of working with a fixed reference vector $\mathbf{g}$ for all $\mathbf{x} \in L(\mathbf{y})$.

Proposition 3. Let $\mathbf{g}=\left(g_{1}, \ldots, g_{m}\right) \in R_{+}^{m}, \mathbf{g} \neq \mathbf{0}$, be a vector nondependent on $\mathbf{x}$. Let $\mathbf{x}_{A} \in L(\mathbf{y})$ and $\mathbf{x}_{B} \in \operatorname{IsoqL}(\mathbf{y})$ such that $\mathbf{x}_{B}=$ $\mathbf{x}_{A}-\beta \mathbf{g}$ with $\beta=\vec{D}_{i}\left(\mathbf{x}_{A}, \mathbf{y} ; \mathbf{g}\right)$ and $\vec{D}_{i}\left(\mathbf{x}_{B}, \mathbf{y} ; \mathbf{g}\right)=0$. Then, $D A I_{A}(\mathbf{g})=$ $D A I_{B}(\mathbf{g})$.

Proof. $D A I_{A}(\mathbf{g})=\frac{\sum_{i=1}^{m} w_{i}\left(x_{A i}-\vec{D}_{i}\left(\mathbf{x}_{A}, \mathbf{y} ; \mathbf{g}\right) g_{i}\right)-C(\mathbf{y}, \mathbf{w})}{\sum_{i=1}^{m} w_{i} g_{i}}=\frac{\sum_{i=1}^{m} w_{i} x_{B i}-C(\mathbf{y}, \mathbf{w})}{\sum_{i=1}^{m} w_{i} g_{i}}$, whereas $\quad D_{A}(\mathbf{g})=\frac{\sum_{i=1}^{m} w_{i}\left(x_{B i}-\vec{D}_{i}\left(\mathbf{x}_{B}, \mathbf{y} \mathbf{g}\right) g_{i}\right)-C(\mathbf{y}, \mathbf{w})}{\sum_{i=1}^{m} w_{i} g_{i}}=\frac{\sum_{i=1}^{m} w_{i} x_{B i}-C(\mathbf{y}, \mathbf{w})}{\sum_{i=1}^{m} w_{i} g_{i}}$, where the last equality is true because $\vec{D}_{i}\left(\mathbf{x}_{B}, \mathbf{y} ; \mathbf{g}\right)=0$. In this way, $D A I_{A}(\mathbf{g})=D A I_{B}(\mathbf{g})$.
However, when $\mathbf{g}=\mathbf{x}$, we have that $D A I_{A}\left(\mathbf{x}_{A}\right)=$ $\frac{\sum_{i=1}^{m} w_{i}\left(x_{A i}-\vec{D}_{i}\left(\mathbf{x}_{A}, \mathbf{y} ; \mathbf{x}_{A}\right) x_{A i}\right)-C(\mathbf{y}, \mathbf{w})}{\sum_{i=1}^{m} w_{i} x_{A i}}=\frac{\sum_{i=1}^{m} w_{i} x_{B i}-C(\mathbf{y}, \mathbf{w})}{\sum_{i=1}^{m} w_{i} x_{A i}}$ and $D_{B} I_{B}\left(\mathbf{x}_{B}\right)=$ $\frac{\sum_{i=1}^{m} w_{i}\left(x_{B i}-\vec{D}_{i}\left(\mathbf{x}_{B}, \mathbf{y} ; \mathbf{x}_{B}\right) x_{B i}\right)-C(\mathbf{y}, \mathbf{w})}{\sum_{i=1}^{m} w_{i} x_{B i}}=\frac{\sum_{i=1}^{m} w_{i} x_{B i}-C(\mathbf{y}, \mathbf{w})}{\sum_{i=1}^{m} w_{i} x_{B i}}$. Therefore, numerators match but denominators are not equal, normalizing the difference between optimal cost and observed cost at $\mathbf{x}_{B}$, the projection point, by a different numeraire. Of course, if the numeraire were the observed cost at $\mathbf{x}_{B}$ even when the point $\mathbf{x}_{A}$ is evaluated, then the allocative inefficiency based on the directional distance function would coincide with the allocative inefficiency related to the Farrell's approach, i.e. $D A I_{A}=A I_{A}$, since, by Proposition 2, $D A I_{A}=\frac{\sum_{i=1}^{m} w_{i} x_{B i}-C(\mathbf{y}, \mathbf{w})}{\sum_{i=1}^{m} w_{i} x_{B i}}=1-\frac{C(\mathbf{y}, \mathbf{w})}{\sum_{i=1}^{m} w_{i} x_{B i}}=1-A E_{B}=1-A E_{A}=A I_{A}$. Unfortunately, this is not the case.

By duality theory (Färe \& Primont, 1995), both approaches are well-grounded. Nevertheless, one might ask whether the existing multiplicative or additive approaches support the above results 
regarding allocative efficiency of technically inefficient firms. In the case of Farrell, as far as we are aware, there is no alternative approach for decomposing cost efficiency that, at the same time, encompasses the Shephard input distance function. In contrast, Briec and Lesourd (1999) introduced the family of Hölder distance functions that has, at least, one element in common with the directional distance functions. In particular, both distances match when the Hölder distance function is linked to the $\ell_{\infty}$ metric and the directional distance function is associated with $\mathbf{g}=1$. Going into detail, Proposition 4.1 in Briec and Lesourd (1999) establishes the following relationship.

$D_{i}^{p}(\mathbf{x}, \mathbf{y})=\inf \left\{\sum_{i=1}^{m} w_{i}^{\prime} x_{i}-C\left(\mathbf{y}, \mathbf{w}^{\prime}\right):\left\|\mathbf{w}^{\prime}\right\|_{q} \geq 1\right\}$,

where $D_{i}^{p}(\mathbf{x}, \mathbf{y}):=\inf \left\{\left\|\mathbf{x}-\mathbf{x}^{\prime}\right\|_{p}: \mathbf{x}^{\prime} \in \operatorname{IsoqL}(\mathbf{y})\right\}$ and $\frac{1}{p}+\frac{1}{q}=1$.

From (5), and invoking that the cost function is homogeneous of degree +1 , we have that $D_{i}^{p}(\mathbf{x}, \mathbf{y}) \leq \sum_{i=1}^{m}\left(\frac{w_{i}}{\|\mathbf{w}\|_{q}}\right) x_{i}-$ $C\left(\mathbf{y}, \mathbf{w} /\|\mathbf{w}\|_{q}\right)=\frac{\sum_{i=1}^{m} w_{i} x_{i}-C(\mathbf{y}, \mathbf{w})}{\|\mathbf{w}\|_{q}}$. In this way, we get the following decomposition of cost inefficiency related to the Hölder distance functions:

$$
\underbrace{\frac{\sum_{i=1}^{m} w_{i} x_{i}-C(\mathbf{y}, \mathbf{w})}{\|\mathbf{w}\|_{q}}}_{H C I(p)}=\underbrace{D_{i}^{p}(\mathbf{x}, \mathbf{y})}_{H T I(p)}+H A I(p),
$$

where the component $\operatorname{HAI}(p)$ measures allocative inefficiency in an additive way.

As we mentioned before, the Hölder distance functions and the directional distance functions are related for the specific case $p=\infty$. In this context, $D_{i}^{\infty}(\mathbf{x}, \mathbf{y})=\vec{D}_{i}(\mathbf{x}, \mathbf{y} ; \mathbf{g})$ for $\mathbf{g}=1$. So, $\operatorname{HAI}(\infty)=\frac{\sum_{i=1}^{m} w_{i} x_{i}-C(\mathbf{y}, \mathbf{w})}{\sum_{i=1}^{m} w_{i}}-D_{i}^{1}(\mathbf{x}, \mathbf{y})=\operatorname{DAI}(\mathbf{1})$ since $q=1$. Hence, the allocative inefficiency term associated with the Hölder distance functions and that derived from the directional distance functions coincide, both obtained from an additive decomposition. This result endows the additive decomposition of cost inefficiency based on the DDF and duality with greater consistency, if at all possible.

Regarding the properties of the input directional distance function, one of great interest is the flexibility of the reference vector when assessing cost inefficiency, departing from the usual direction $\mathbf{g}=\mathbf{x}$. The choice of a different directional vector can provide some guidance on alternative options: Setting the normalization constraint to $\sum_{i=1}^{m} w_{i} g_{i}=1$ ensures that cost inefficiency is measured in monetary values, which is an attractive feature, while the flexibility of $\mathbf{g}$ can accommodate any orientation. For example, it is possible to choose a directional vector that rescales the observed input vector $\mathbf{x}$ so that the previous constraint is satisfied (as in the Farrell approach), or a vector that is neutral with respect to the orientation, by weighting all inputs equally - see Zofío, Pastor, and Aparicio (2013). In the former case, the cost inefficiency of firm $B$ in Fig. 1 is 3 monetary units, with technical and allocative inefficiencies equal to 2.5 and 0.5 monetary units, respectively. As a result, and depending on their choice of analytical model, researchers face alternative measures for the allocative (in)efficiency terms $A E$ and $D A I$ and, by extension, the complete measurement and decomposition of cost (in)efficiency.

\section{Conclusions}

It can be concluded from our analysis that practitioners must keep in mind that, in contrast to what has been commonly accepted, the choice between the traditional Farrell approach or the directional input distance function approach for decomposing cost efficiency with $\mathbf{g}=\mathbf{x}$ is quite relevant, since they are not equivalent, yielding alternative decompositions unless the evaluated firm is technically efficient. Indeed, one may believe that the $D D F$ approach underestimates allocative inefficiency, or that the Farrell approach overestimates it, depending on the reference framework.

However, by systematically exploring the difference between both approaches, it is relatively easy to gauge the discrepancy that exists between them, depending on the specific values that are obtained for the allocative and efficiency terms. For example, using the average values for all firms included in an empirical study, it is possible to determine the disparity between the multiplicative and additive approaches, knowing that the higher these values, the lower the allocative inefficiency discrepancy between the two.

In this respect, the only way to address the choice for a specific approach hinges upon the information that each one provides, as it must be acknowledged that both decompositions cannot be reconciled given their multiplicative and additive nature, representing two alternative ways to measure allocative efficiency. In this way, practitioners must weigh the trade-offs between both approaches and the different, but complementary information, which they provide, depending on their choice of analytical framework for economic efficiency analysis.

\section{Acknowledgments}

The authors would like to thank three anonymous referees for their helpful comments and suggestions and acknowledge the financial support from the Spanish Ministry of Economy and Competitiveness under Grant MTM2013-43903-P.

\section{References}

Aparicio, J., Borras, F., Pastor, J. T., \& Vidal, F. (2013a). Accounting for slacks to measure and decompose revenue efficiency in the Spanish designation of origin wines with DEA. European Journal of Operational Research, 231, 443-451.

Aparicio, J., Pastor, J. T., \& Ray, S. C. (2013b). An overall measure of technical inefficiency at the firm and at the industry level: The 'lost profit on outlay'. European Journal of Operational Research, 226, 154-162.

Aparicio, J., Borras, F., Pastor, J. T., \& Vidal, F. (2015a). Measuring and decomposing firm's revenue and cost efficiency: The Russell measures revisited. International Journal of Production Economics, 165, 19-28.

Aparicio, J., Pastor, J. T., \& Zofio, J. L. (2015b). How to properly decompose economic efficiency using technical and allocative criteria with non-homothetic DEA technologies. European Journal of Operational Research, 240, 882-891.

Balk, B. M., Färe, R., Grosskopf, S., \& Margaritis, D. (2008). Exact relations between Luenberger productivity indicators and Malmquist productivity indexes. Economic Theory, 35, 187-190.

Boussemart, J. P., Briec, W., Kerstens, K., \& Poutineau, J. C. (2003). Luenberger and Malmquist productivity indices: Theoretical comparisons and empirical illustration. Bulletin of Economic Research, 55(4), 391-405.

Briec, W. (1997). Minimum distance to the complement of a convex set: Duality result. Journal of Optimization Theory and Applications, 93(2), 301-319.

Briec, W. (1998). Hölder distance function and measurement of technical efficiency. Journal of Productivity Analysis, 11, 111-131.

Briec, W., \& Lesourd, J. B. (1999). Metric distance function and profit: Some duality results. Journal of Optimization Theory and Applications, 101(1), 15-33.

Briec, W., Kerstens, K., \& Peypoch, N. (2012). Exact relations between four definitions of productivity indices and indicators. Bulleting of Economic Research, 64(2), 265-274

Caves, D., Christensen, L., \& Diewert, W. (1982). The economic theory of index numbers and the measurement of input, output and productivity. Econometrica, 50(6) 1393-1414.

Chambers, R. G., Chung, Y., \& Färe, R. (1996a). Benefit and distance functions. Journal of Economic Theory, 70, 407-419.

Chambers, R. G., Färe, R., \& Grosskopf, S. (1996b). Productivity growth in APEC countries. Pacific Economic Review, 1, 181-190.

Chambers, R. G., Chung, Y., \& Färe, R. (1998). Profit, directional distance functions and Nerlovian efficiency. Journal of Optimization Theory and Applications, 98(2), $351-364$

Chavas, J. P., \& Cox, T. M. (1999). A generalized distance function and the analysis of production efficiency. Southern Economic Journal, 66(2), 295-318.

Cooper, W. W., Pastor, J. T., Aparicio, J., \& Borras, F. (2011). Decomposing profit inefficiency in DEA through the weighted additive model. European Journal of Operational Research, 212(2), 411-416.

Debreu, G. (1951). The coefficient of resource utilization. Econometrica, 19(3) 273-292. 
Färe, R., \& Primont, D. (1995). Multi-output production and duality: Theory and applications. Kluwer Academic.

Färe, R., \& Grosskopf, S. (1997). Profit efficiency, Farrell decomposition and the Mahler inequality. Economics Letters, 57, 283-287.

Färe, R., \& Grosskopf, S. (2000). Theory and application of directional distance functions. Journal of Productivity Analysis, 13(2), 93-103.

Färe, R., Grosskopf, S., \& Zaim, O. (2002). Hyperbolic efficiency and return to the dollar. European Journal of Operational Research, 136, 671-679.

Färe, R., \& Grosskopf, S. (2003). New directions: Efficiency and productivity. Kluwer Academic Publishers.

Färe, R., Fukuyama, H., Grosskopf, S., \& Zelenyuk, V. (2015). Decomposing profit efficiency using a slack-based directional distance function. European Journal of Operational Research, 247(1), 335-337.
Farrell, M. J. (1957). The measurement of productive efficiency. Journal of the Royal Statistical Society: Series A (General), 120, 253-281.

Luenberger, D. G. (1992). New optimality principles for economic efficiency and equilibrium. Journal of Optimization Theory and Applications, 75(2), 221-264.

Shephard, R. W. (1953). Cost and production functions. Princeton Univ. Press.

Zalinescu, C. (2010). On the duality between the profit and the indirect distance functions in production theory. European Journal of Operational Research, 207, $30-36$.

Zofío, J. L., Pastor, J. T., \& Aparicio, J. (2013). The directional profit efficiency measure: On why profit inefficiency is either technical or allocative. Journal of Productivity Analysis, 40, 257-266. 